Artigo original

\title{
REFLEXIONES EL ARTE, LA EDUCACIÓN ARTÍSTICA Y SU SITUACIÓN EN BOA VISTA - RR
}

\author{
Rosangela DUARTE ${ }^{1}$
}

RESUMEN: El presente trabajo tiene la finalidad de identificar los factores objetivos y subjetivos que interfieren en el desarrollo con calidad de la Educación Artística. Para hablar de los factores que comprometan la calidad de la enseñanza del Arte en la escuela se debe tener conciencia de las diferentes posiciones que sobre el arte y la educación influyen en la selección de líneas teórico metodológicas. Entre los factores discutidos resaltamos la busqueda del arte en captar, fijar y exaltar las modalidades del hombre; el arte se inserta en la expresión personal de valores, sentimientos y significaciones y es un factor de agilización de nuestra imaginación, ampliando los limites que le imponen cotidianamente la intelección. Los docentes que actúan en las escuelas de Boa Vista trabajan en condiciones desfavorables y deben buscar conocimientos con el fin de transmitir seguridad a sus alumnos, desarrollando su creatividad y obedeciendo al compromiso de alcanzar la realización plena e integral del hombre.

Palabras-claves: reflexión; Arte; Educación Artística; calidad.

RESUMO: Este trabalho tem a finalidade de identificar os fatores objetivos e subjetivos que interferem no desenvolvimento com qualidade da Educação Artística. Para falar dos fatores que comprometam a qualidade do ensino da Arte na escola se deve ter consciência das diferentes posições que sobre a Arte e a Educação influem na seleção de linhas teórico-metodológicas. Entre os fatores discutidos ressaltamos a busca da Arte em captar, fixar e exaltar modalidades do homem. A Arte se inserta na expressão pessoal de valores, sentimentos e significações. É um fator de agilização de nossa imaginação, ampliando os limites que impõem cotidianamente 0 intelecto. Os docentes que atuam nas escolas de Boa Vista trabalham em condições desfavoráveis e devem buscar conhecimentos a fim de transmitir segurança a seus alunos, desenvolvendo sua criatividade e obedecendo ao compromisso de alcançar a realização plena e integral do homem.

Palavras-chaves: reflexão; Arte; Educação Artística; qualidade.

${ }^{1}$ Professora, Coordenadora da ABEM/RR, Mestra em Educação Superior pela Universidade de Matanzas-Cuba

Bol. Mus. Integrado de Roraima, Boa Vista 6:37-44. 2000[2007] 


\section{INTROCUCIÓN}

El presente trabajo tiene la finalidad de identificar los factores objetivos y subjetivos que interfieren directamente en el desarrollo con calidad de la Educación Artística. Bajo la perspectiva de la dialéctica de Marx, observamos las contradicciones de los conceptos que rigen la educación, siendo esta considerada por el Programa de las Naciones Unidas para el Desarrollo - PNUD un índice del Desarrollo Humano, que muestra la realidad como es desarrollada dentro de los países Latinos Americanos, como el Brasil.

El Desarrollo Humano es un proceso mediante el cual se aumentan las oportunidades del hombre, no solamente buscando el crecimiento económico, sino también el afloramiento de sus capacidades, centralizando sus necesidades, aspiraciones y opciones, en las actividades del desarrollo.

El hombre pasa a ser considerado el objeto y sujeto del desarrollo, atribuyéndole la posibilidad y necesidad de participar activamente en los procesos de ampliación de sus propias oportunidades.

La educación para un mundo en "movimiento" debe ser una educación plena, donde la escuela ejerza su papel integrador ante la realidad conocida por la comunidad en la cual esté insertada. Pensar en educación plena es pensar en "educación con calidad".

Por lo tanto, concordando con el pensamiento de Pedro Demo, definimos calidad como competencia humana, construcción humana, sobrepasando la cuestión de los medios y de los fines. Nos hacemos eco al desafío del desarrollo humano sustentado, que encuentra en la educación y en el conocimiento su estrategia primordial.El mecanismo del conocimiento humano es un juego dialéctico entre 10 que es sentido (vivido) y lo que es simbolizado (transformado en palabras u otros símbolos).

Siendo esto así, la Educación Artística no significa solamente la inclusión del arte en los currículos de las escuelas. La Educación Artística tiene que ver con un modelo educacional fundado en la construcción de un sentido personal para la vida que sea propio de cada educando.

No se tiene la intención de afirmar que sobre el arte reposan todas las soluciones para los problemas creados por nuestra "dividida" civilización y su educación impuesta. Sólo se afirma que el arte es un factor importante para la vida humana en la medida que permite el acceso a las dimensiones no reveladas por la lógica y por el pensamiento discursivo. A través del arte se opera la educación de los sentimientos, auxiliando dialécticamente en la educación del pensamiento lógico, como afirma Rubens Alves: "a menos que abandonemos la lógica dominante de este presente orden de las cosas y nos hagamos creadores, nunca viviremos lo bastante para mirar el futuro. Estaremos condenados a la extinción. La extinción decretada por la imposibilidad del dominio de la lógica racionalista sobre la lógica del corazón".

\section{El arte como forma de actividad estética.}

La actividad estética, según Aranha \& Martins, representa una de las formas de apropiación práctico espiritual de la realidad por el hombre, con lo cual este último incorpora un nuevo ingrediente al mundo que lo rodea, contribuyendo a la humanización de su medio.

Como toda forma de actividad humana, la actividad estética posee 
un carácter material (práctico) y uno ideal (conciencia estética). La práctica estética corresponde al conjunto de acciones de carácter material que tiene lugar en el proceso de ínterrelación estética del hombre con la realidad. Se manifiesta en la transformación del objeto según los gustos y necesidades estéticas del sujeto.

Del mismo modo, la conciencia estética constituye el reflejo ideal de las propiedades de la realidad y de la periodicidad con que transcurre la relación estética del hombre con la realidad.

El vínculo de lo material y de lo ideal en la actividad estética tiene lugar en el contexto de la interacción del objeto y del sujeto.

El sujeto de la actividad estética es aquel individuo o sociedad que ejerce una acción de orden estética sobre un fenómeno o proceso determinado.

El objeto de la actividad estética es aquella parte o ángulo de la realidad natural o social, incluyendo el hombre, que recibe el resultado de la acción material - ideal orientada a satisfacer las necesidades estéticas del sujeto. Siendo así, la actividad estética se encuentra vinculada a las formas fundamentales de la actividad humana.La actividad estética se realiza, fundamentalmente, a través del arte.

El arte "se caracteriza por el descubrimiento de lo racional a través de lo emocional. El arte constituye un modo de captar y materializar los sentimientos $y$ emociones humanas donde se muestra la existencia subjetiva íntima del hombre mediante formas sensorialmente perceptibles que nos permiten oír y ver las mismas."(Serpa, 1996, p.130).El arte es un producto de la actividad del hombre considerada como un proceso que se renueva y se realiza permanentemente, dentro de un contexto histórico y social. Dentro de este contexto, toda actividad artística se encuentra constituida por un objeto y un sujeto.

El objeto del arte es una faceta de la realidad que posee determinado valor humano y social y que es captado estéticamente a través de los medios artísticos. A su vez, el sujeto del arte puede ser el individuo o la comunidad que participa o vive, en cualquiera de los momentos, de la actividad artística (el artista, el publico, el crítico de arte, y otros).

Podemos decir que el mediador entre el sujeto y el objeto del arte es la propia obra de arte.

Corroborando al pensamiento de la filósofa Marilena Chauí, la correlación del objeto y el sujeto del arte trae consigo el vínculo de lo objetivo y subjetivo en la actividad artística.Lo objetivo se encuentra asociado a los materiales de los cuales la obra es hecha, tanto en la producción como en el consumo de materiales que no depende de la conciencia y tampoco de la voluntad de los sujetos que de ella participan.

Lo subjetivo viene de la interpretación que es dada a la obra, por parte del sujeto que la crea o que la percibe, a partir de sus conocimientos, se relaciona directamente al hecho emocional en el arte.

"A partir de la imbricación de lo objetivo y lo subjetivo en el arte se logra precisamente que el mismo opere como una de las vías que posee el hombre tanto para sensibilizar las ideas como para racionalizar los sentimientos, lo que también se refleja en el poder sugestivo del mismo." (serpa, 1996, p. 224 ).

\section{El Arte y la Educación.}

Para hablar de los factores que comprometan la calidad de la enseñanza del Arte en la escuela se debe tener conciencia de las 
diferentes posiciones que sobre el arte y la educación en la escuela influyen en la selección de líneas teórico metodológicas.

"Arte es calidad y ejercita nuestra habilidad de juzgar y de formar significados que exceden nuestra capacidad de hablar en palabras." (Barbosa, 1994,p.4)

El propio concepto del Arte ha sido objeto de diversas interpretaciones: arte como técnica, diversión, materiales artísticos, procesos intuitivos, expresiones, lenguaje, etc.

Siendo así, la concepción del arte que puede auxiliar en la fundamentación de una propuesta de la enseñanza y aprendizaje artístico, estético y que atienda esta variación conceptual, es la que conduce a una articulación del hacer, del representar y del decir. "El hacer técnico inventivo, el representar con imaginación el mundo de la naturaleza y de la cultura y la expresión de la síntesis de sentimientos están incorporados en las acciones del productor de la obra artística en la propia obra de arte, en el proceso de su presentación a la sociedad $y$ en los actos de los espectadores." (Fusari y Ferraz, $1992, \mathrm{p} .19)$.

Todos esos elementos movilizadores del arte deben mantenerse presentes como un saber a ser aprendido a lo largo del proceso de la escuela. De este modo, la enseñanza del arte como disciplina, garantiza que los estudiantes conozcan y vivencien aspectos técnicos, inventivos, representativos y expresivos en la música, las artes visuales y la danza.El maestro, conjuntamente con sus alumnos, deben incorporar acciones como: ver, oír, pensar, sentir, descubrir, decir y hacer, a partir de los elementos de la naturaleza y de la cultura, analizando, reflejando, formando y transformándolos.
La interacción del Arte con las otras disciplinas, dentro del contexto de la educación escolar, contribuye a la preparación de los individuos para que perciban mejor el mundo en que viven.

La educación para Pedro Demo, ha sido el término escogido para designar calidad, por una serie de razones:

a) Como instrumento, señala la construcción del conocimiento y, como fin, la preocupación en torno a la humanización de la realidad y de la vida;

b) Como expediente formativo, primordial de las nuevas generaciones, representa un procedimiento de los mas pertinentes en términos de calificar a la población, tanto para crear los medios como para alcanzar los fines;

c) Principalmente, estando en la base de la formación del sujeto histórico, crítico y creativo, la educación cumple la estrategia mas decisiva de propicia la oportunidad.

Utilizase el concepto de "educación de calidad" para acentuar su compromiso constructivo del conocimiento. No hay como llegar a la calidad sin educación, así como no será educación aquella que no se destine a formar el sujeto histórico, crítico y creativo.

La Organización de las Naciones Unidas - ONU considera la educación como un índice de desarrollo humano, por ser condición necesaria para conocer y propiciar la oportunidad. Sin conciencia crítica, conocimiento y participación no es viable el desarrollo humano, porque la población ni siquiera se descubre como oportunidad. Además de esto, cabe a cada sociedad construir su proyecto de desarrollo y dotarlo de la cualidad histórica posible. Más, "no es posible una educación intelectual, formal o informal, de élite o popular, sin arte, porque 
es imposible el desarrollo integral de la inteligencia sin el desarrollo del pensamiento divergente, del pensamiento visual y del conocimiento presentacional que caracterizan al arte." (Barbosa, $1994, \mathrm{p} .5$ )

\section{Factores objetivos y subjetivos de} la Educación Artística.

Siendo el arte la concretización de los sentimientos en formas expresivas ella se constituye en un medio de acceso a las dimensiones humanas no posibles de simbolización conceptual.

"Arte - Educación (...) quiere significar una educación que tenga al arte como una de sus principales aliadas. Una educación que permita una mayor sensibilidad para con el mundo alrededor de cada uno de nosotros." (Duarte Jr, 1991).

Es a través de la educación artística que el hombre es preparado para apreciar y disfrutar las obras de arte, adquiriendo conocimientos que lo conducirán a captar la naturaleza esencial del mundo que lo rodea y sus más profundas significaciones humanas.

El lenguaje proporciona el encuentro del hombre con el mundo y lo fragmenta en conceptos y relaciones, que se ofrecen a la razón, al pensamiento. El arte procura revivir en nosotros este encuentro, este "primer mirar" sobre las cosas. A través del arte somos llevados a conocer mejor nuestra experiencia y sentimientos en aquello que escapa a la linealidad del lenguaje.

Es importante considerar que el arte busca en sí mismo captar, fijar y exaltar las cualidades del hombre como tal. Sintetiza las mejores características y valores de su esencia, comportamiento e interrelación con los demás hombres, con independencia de las condiciones o épocas históricas en que desarrolle su actividad. Con este componente universal del arte se pueden admirar obras creadas hace mucho tiempo y que hoy aún despiertan los mismos sentimientos en los espectadores.

La educación, especialmente en el campo artístico no es una actividad "neutra" como las ciencias exactas, sino que se inserta en la expresión personal de valores, sentimientos y significaciones. Se despierta la atención de cada individuo por su manera particular de sentir, sobre la cual se elaboran todos los otros procesos racionales. En el momento en que el individuo entre en contacto con la obra de arte, este disfrutará de su valor estético que está relacionado tanto con el placer que nos proporciona, como con los conocimientos que nos brinda y las actitudes positivas que nos provoca.

"La actividad artística forma y organiza la esfera de la percepción estética del mundo por el hombre, es decir, de la sensibilidad específicamente humana donde mediante la educación de los sentidos se logra transgredir la simple observación y se alcanza la contemplación y disfrute consciente, racional e intelectualizado de la realidad, desarrollándose con ello el sentimiento de la belleza." (Serpo, 1996, p.230).

El arte es aún un factor de agilización de nuestra imaginación, pues en la experiencia estética la imaginación amplía los limites que le imponen cotidianamente la intelección. Por el arte la imaginación es invitada a actuar, rompiendo el estrecho espacio que lo cotidiano le reserva. Son nuestros sueños y proyectos para mover el mundo. El no "tener" lo que aún el hombre no ha conseguido es lo que le hace ir a luchar para alterar la realidad.

Al proponer nuevas "realidades posibles", el arte permite que 
tomemos conciencia del actual estado del mundo humano y se perciba lo distante que se encuentra nuestra sociedad de un estado más equilibrado, lúdico y estético.

El arte nos lleva a conocer aquello que no tenemos oportunidad de experimentar en nuestra vida cotidiana. Y esto es básico para que se puedan comprender las experiencias vividas por otros hombres.

Según las palabras del filósofo alemán Ernst Fischer: "El deseo del hombre de desarrollar y completar indica que él es mas que un individuo. Siente que solo puede alcanzar la plenitud si se apodera de las experiencias ajenas que potencialmente le conciernen, que podrían ser de él. Y lo que el hombre siente como potencialmente suyo incluye todo aquello que la humanidad, como un todo, es capaz. El arte es el medio indispensable para esta unión del individuo con el todo; refleja la infinita capacidad humana para la asociación, para la circulación de experiencias e ideas." (Ficher, $1976, \mathrm{p} .13$ )

Así, esos son algunos de los factores educativos centralizados en la educación artística. Estos son los fundamentos filosóficos que sirven de base para la utilización del arte como vehículo educacional.

\section{El arte en la Educación en Boa vista.}

A partir de lo que fue expuesto, se puede sentir la necesidad de verificar las condiciones reales de la enseñanza del arte en las escuelas de Boa Vista - RR.

Partiendo de la experiencia profesional como docente, hemos constatado muchas dificultades relacionadas con la formación. Siendo Roraima un Estado nuevo y geográficamente aislado de los grandes centros, el reciclaje de los maestros que aquí llegan se torna escaso y muy caro. Con la dependencia económica totalmente centrada en el Gobierno Federal y Estadual, la iniciativa para patrocinios de cursos y congresos prácticamente es inexistente.

En el municipio de Boa Vista existen unidades escolares particulares y públicas, (siendo esta última la gran mayoría) y como es la capital del Estado, en ella se concentra el mayor número de profesores. Dentro del cuadro de docentes de cada escuela existen profesores federales, estatales y contratados por la lista especial. Por el aislamiento geográfico en que se encuentra el Estado de Roraima del resto del país, la selección para la contratación de profesores nunca fue rígida. Siempre fue considerado como prioridad la atención a las necesidades educacionales con los profesionales ya residentes en el Estado, aún cuando estos no tengan una formación especifica para el magisterio.

Se puede constatar que los docentes que actúan como profesores de educación artística tienen formación en las áreas más diversas como: matemática, historia, física, teología, y otras. Esto se da por el hecho de que el profesor necesita tener una carga horaria mínima de 25 horas en las clases, para recibir la complementación salarial integral ofrecida por FUNDEF.

Con la limitación de la formación del docente, del espacio físico y de los materiales didácticos específicos para las clases de educación artística, se observa que los programas del arte desarrollados en las escuelas se resumen en diseños, pinturas, teatro, y trabajos con "sucata". La mayoría de las actividades están dirigidas hacia las fechas conmemorativas de la escuela, donde los alumnos presentan una poesía, una danza folklórica o una música cantada. La fundamentación teórica del arte, su contextualización en 
el tiempo y en el espacio, no es repasada por los alumnos.

Según resultados de pesquisa recientes "los profesores consideran que la aceptación de las clases de arte por los alumnos es buena y siempre se presenta un mayor aprovechamiento cuando los alumnos tienen la oportunidad de trabajar en talleres. Por esto reivindican un espacio propio y único para impartir sus clases". (Duarte, 1997.

Otro factor importante es la falta de reconocimiento de la importancia del trabajo realizado por el docente de artes por los colegas de otras disciplinas. Pocos piensan que el arte tiene como función proporcionar que el individuo se desarrolle con una visión crítica y creativa. Y que este "hacer" cultural se refleja directamente en todas las áreas, f a voreciendo

interdisciplinariedad.

El profesor de arte, muchas veces se siente sólo frente a las limitaciones que encuentra para superar las dificultades con el fin de mejorar la calidad de su trabajo. Para desarrollar un programa de calidad, con fundamentación teórico y metodológica, el profesor necesita tener más apoyo e incentivo, principalmente en lo que respecta a su formación.

\section{Conclusión.}

La formación del profesor de Educación artística en Boa Vista $\mathrm{RR}$ es muy deficitaria por el hecho de que la mayoría no posee una formación universitaria específica en esa área.

Los docentes que actúan hoy en las escuelas trabajan en condiciones desfavorables en relación a espacio físico y a los materiales didácticos específicos disponibles, para la realización de un programa de Educación artística rico y creativo.

La educación artística es la oportunidad para que cada uno exprese aquello que siente y percibe. A partir de esta expresión personal es que se puede llegar a aprender cualquier tipo de conocimiento impartido por los otros. El docente no puede negar esta oportunidad a sus alumnos y al mismo tiempo no cuenta con una formación al nivel de desarrollar un programa que venga de acuerdo a sus necesidades.

El profesor de arte debe buscar conocimientos con el fin de transmitir seguridad a sus alumnos, incentivando la búsqueda del desarrollo de la creatividad, obedeciendo al compromiso de alcanzar la realización plena e integral del hombre.

sugerimos, como acción inmediata, que haya una preocupación por parte de la Secretaria Estadual de la Educación, en una acción conjunta con la UFRR, para que sean ofrecidos cursos de extensión, buscando la capacitación de estos profesores que están actuando en las escuelas de Boa Vista.

Se reafirma la necesidad de implantar un curso de Licenciatura en las áreas de Artes (música, teatro, artes plásticas y Danza), a través de la UFRR, o por otra Universidad como curso secuencial, lo cual actualmente viene siendo muy divulgado por el Ministerio de la Educación - MEC.

\section{Bibliografia :}

ARANHA, $M^{a}$ Lucia de Arruda y Martins, $\mathrm{M}^{\mathrm{a}}$ Helena Pires. Filosofando Introducción a la filosofía. São Paulo : Moderna, 1993.

CHAUI, Marilena. Convite à Filosofia.são Paulo: Ática, 1995. DEMO, Pedro. Educação e qualidade. Campinas: Papirus, 1996.

DUARTE JR., João Francisco. Porque arte 
- educação? Campinas: Papirus, 1991.

DUARTE, Rosangela. O professor e sua Formação: uma visão contemporânea do professor de artes em Boa Vista - RR. Rio de Janeiro, 1997.

Educación e Desarrollo. 2000. Selección de Lecturas. Universidad de Matanzas "Camilo Cienfuegos". Matanzas - Cuba, p.01-22.

FISCHER, Ernst. A necessidade da arte. Rio de Janeiro: Guanabara, 1987.
MARINS, José Salgado. Preparação à filosofia. Porto Alegre : Globo, 1973 .

POPPER, Karl R. Conjecturas e refutações. Brasilia: Ed. Universidade de Brasilia, 1982.

PORCHER, Louis. Educação artística, luxo ou necessidade? São Paulo: Summus, 1982.

SERPA, Gerardo R. La actividad humana $y$ sus formas fundamentales. Cuba: Universidad. 